Case Reports
in Dermatology
Case Rep Dermatol 2021;13:134-140

DOI: $10.1159 / 000512869$

Published online: February 18, 2021

(c) 2021 The Author(s)

Published by S. Karger AG, Basel

www.karger.com/cde

This article is licensed under the Creative Commons Attribution-NonCommercial 4.0 International License (CC BY-NC) (http://www.karger.com/Services/OpenAccessLicense). Usage and distribution for commercial purposes requires written permission.

\author{
Single Case
}

\title{
Recurrent Erythema Annulare Centrifugum due to Influenza Type A
}

\author{
Luca Ena ${ }^{a, b}$ Vittorio Mazzarello ${ }^{b}$ Marco Ferrarib, ${ }^{b}$ Pasquale Ena ${ }^{b, d}$ \\ aDermatology Unit of Asti's Hospital, Cardinal Massaia Hospital, Asti, Italy; \\ bSkinlab, Department of Biomedical Sciences, University of Sassari, Sassari, Italy; \\ 'Department of Medicine, Surgery and Health Sciences, University of Trieste, Trieste, Italy; \\ dPrivate Practitioner, Sassari, Italy
}

\section{Keywords}

Erythema annulare centrifugum · Influenza - Paracetamol $\cdot$ Cytomegalovirus $\cdot$ Cell-mediate immunity

\begin{abstract}
Erythema annulare centrifugum (EAC) is a rare erythema characterized by erythematous and urticarial papules or annular plaques that enlarges centrifugally. The lesions usually involve the thighs and the legs. Several disorders are occasionally associated with EAC, infections, including mycoses, bacteria, or viruses and drugs have also been regarded as possible causes of this eruption. We present a 42 -year-old dark-skinned woman affected by recurrent EAC that appeared secondary to influenza type A (H1N1). Histopathology showed a superficial form of EAC. In our case, a previous cytomegalovirus and Epstein-Barr virus (EBV) infection were identified and no underlying other diseases were found. Clarithromycin with calcipotriol betamethasone treatment was temporarily efficacious. In the last 3 years, the lesions started to appear every 2 weeks and tended to regress with local treatment after a variable period. We believe that the latent cytomegalovirus and the reactivity induced by EBV combined with influenza can determine, in our case, a cell mediate cutaneous immune response, which leads to the peculiar inflammatory disease known as EAC.

(C) 2021 The Author(s)

Published by S. Karger AG, Basel
\end{abstract}

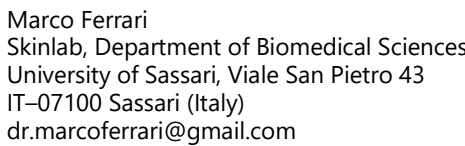




\section{Case Reports in Dermatology}

Case Rep Dermatol 2021;13:134-140

DOI: $10.1159 / 000512869$

(C) 2021 The Author(s). Published by S. Karger AG, Basel www.karger.com/cde

Ena et al.: Recurrent Erythema Annulare Centrifugum due to Influenza Type A

\section{Introduction}

Erythema annulare centrifugum (EAC) is a reactive disorder that appears as urticariallike papules and erythematous plaques with crusts or vesicles that enlarge by peripheral extension with central clearing, resulting in annular or polycyclic appearance. EAC is a relatively rare inflammatory disorder described by Darier in the annular erythema's group. EAC secondary to influenza A in a dark-skinned woman was never described in this condition. Infectious diseases, drug use, systemic diseases, autoimmune disorders, and internal malignancies are occasionally associated [1].

\section{Case Presentation}

A 42-year-old native Indian woman was referred to our department in October 2016 with a 6-month history of a relapsing, mild itchy eruption involving the legs and abdomen. This eruption was concomitant with seasonal influenza (cough, fever, and fatigue), lasting for 6 weeks, treated with paracetamol 1,000 mg/day and sometimes tripolidine. She was otherwise healthy and had no lymphadenopathy. Physical examination revealed several erythematous and violaceus annular plaques, measuring from 2 to $10 \mathrm{~cm}$ in diameter, with a thick central area and a centrifugal spread on forearms, lower abdomen, buttocks, thighs, and lower legs. The lesions were located symmetrically in the inguinal and upper-exterior regions of the thighs. Some of these plaques presented an erythematous edge and peripheral scaling (Fig. 1a, b). Routine blood investigation revealed a moderate neutrophilic leukocytosis $(13.5 \times$ $\left.10^{3} / \mathrm{mm}^{3}: 4.50-12.5\right)$, hypercholesterolemia, and increase of C-reactive protein $(19.64 \mathrm{mg} / \mathrm{L}$ : 0.0-5.0). Serological tests for Borrelia burgdorferi, HIV, hepatitis viruses A, B, C, anti-streptolysin title, and syphilis serology were negative. Furthermore, IgG anti-cytomegalovirus (CMV) (463.0 AU/mL: >15 AU/mL), VCA-EBV IgG Ab, and varicella zoster were positive. IgM anticytomegalovirus, anti-gad, and anti-IA antibodies were negative. Laboratory tests were negative for fungi and bacteria. Stool exam for bacteria and parasites and search of Helicobacter pylori were negative. Thyroid function tests and immunology laboratory test to measure ENA panel and immunoglobulins were negative. Tuberculin test was moderately positive. A chest $\mathrm{X}$-ray and mammogram showed no abnormalities. Gynecological examination, transvaginal ultrasound, and PAP test were negative. Histopathologic examination of a skin biopsy revealed an intense dermal perivascular lymphohistiocytic infiltration with a "sleeve-like" arrangement. There was edema of the papillary dermis, hyperkeratosis, and focal epidermal spongiosis (Fig. 2a, b). Direct skin immunofluorescence test and PCR for CMV-DNA in biopsy specimen results were negative. Clinic-pathological findings were consistent with the diagnosis of a superficial form of EAC. Anti-CMV IgG antibodies remained elevated, and previous therapy with antihistamines and topical corticosteroids was not effective. Treatment with clarithromycin $500 \mathrm{mg} /$ day and topical calcipotriol/betamethasone for 6 weeks resulted in temporary regression of the annular lesions. The course of the disease was chronic and recurrent over 3 years. Annular and discoid-shaped lesions that did not exceed $3 \mathrm{~cm}$ in diameter, on the lower limbs and thighs, reappeared about every 2 weeks during the summer, fall, and winter (Fig. 


\section{Case Reports in Dermatology}

Case Rep Dermatol 2021;13:134-140

DOI: $10.1159 / 000512869$

(C) 2021 The Author(s). Published by S. Karger AG, Basel www.karger.com/cde

Ena et al.: Recurrent Erythema Annulare Centrifugum due to Influenza Type A

1c). These lesions disappeared with the same topical treatment, while similar annular skin changes occurred in the adjacent areas. No relapses occurred after 6 months of follow-up.

\section{Discussion}

The diagnosis of EAC was based on the suggestive figurate erythema and histopathological pattern. This disorder is rare and usually occurs on the proximal extremities, thighs, arms, or trunk and clears up by itself in variable time duration. EAC can mimic nummular eczema, annular granuloma, erythema multiforme, erythema marginatum, cutaneous B-cell lymphoma, annular sarcoidosis, cutaneous lupus erythematosus, erythema gyratum repens, tinea corporis, mycosis fungoides, and figurate psoriasis. A superficial and a deep type histopathologic variant have been described. In the superficial variant of EAC, infiltrates of histiocytes, lymphocytes, and rarely eosinophils are present around vessels of superficial plexus. The infiltrate, with tight aggregate around the vessels, is known as "coat-sleeve" [2]. The etiology and pathogenesis are unknown and this condition is interpreted as a hypersensitivity reaction to different causes, including viral infections. For these reasons, a full physical examination and laboratory investigations should exclude associated malignancies. EAC has been associated with many infections, particularly dermatophytoses, Candida spp., but also viruses (EBV, poxvirus, HIV, and HVZ), parasites, pediculosis pubis, and bacteria [3]. The interaction between latent CMV infection and influenza is not defined. In our case, the subtype A/H1N1 influenza virus may be regarded a possible pathogenic agent in the onset of the eruption. During that time in Italy, this type of virus was mainly responsible for influenza [4], even with serious cases. We also believe that the triggering of the erythematous lesions can be a cutaneous hypersensitivity reaction due to the prolonged action of influenza virus. EAC was associated with herpes zoster, considering this cutaneous eruption is due to an alteration of the local immunity known as Wolf's isotopic response [5]. Varicella zoster DNA was not found in the skin of the described case, suggesting that the EAC took place not as a direct consequence of local viral infection, but through a type IV immune reaction to the herpes virus. This mechanism could explain a similar action with the release of cytokines from the cutaneous immune system stimulated from the influenza virus and, secondly, from CMV. Macular-papular rashes, cutaneous vasculitis, and Gianotti-Crosti syndrome rarely occur in patients with influenza viruses [6]. A pityriasis rosea that developed after the H1N1 vaccination would demonstrate the role of the immune response of the skin to virus antigens [7]. Reactive EAC associated with various infections tends to resolve spontaneously within a few weeks, while in cases with underlying malignancy, the lesions either do not disappear spontaneously or reappear after a short time. However, a patient with a variant form of EAC showed relapse during many years for no apparent reason $[8,9]$. The therapeutic validity of macrolides, especially clarithromycin, was demonstrated in cases of idiopathic EAC [10] and clarithromycin resulted in efficacy also in our challenging case. EAC secondary to influenza A in a dark-skinned woman was never described in this condition. Paracetamol and tripolidine taken during viral infection are not pharmacological concausal molecules [11] like triggering EAC (Table 1). However, we cannot exclude that paracetamol may have had an initial role in the pathogenesis of a superficial 


\section{Case Reports in Dermatology}

perivascular EAC-like dermatitis. In conclusion, in our patient, the genesis and the marked recurrence of EAC may be secondary to influenza and a possible individual predisposition of the dark skin. We believe that the latent cytomegalovirus and reactivity induced by EBV, combined with influenza virus, can determine a cell-mediate immunity response of the skin, related to a molecular change miming viral epitopes. Hypothetically, anti-influenza therapy such as paracetamol can amplify the immune-mediated cellular response to flu viruses. This hypersensitivity reaction leads to the peculiar inflammatory disease known as EAC.

\section{Statement of Ethics}

Written informed consent to publish this case (including images) was obtained from the patient. The research was conducted ethically in accordance with the World Medical Association Declaration of Helsinki.

\section{Conflict of Interest Statement}

The authors have no conflicts of interest to declare. The authors alone are responsible for the content and writing of the paper.

\section{Funding Sources}

The authors did not receive any funding.

\section{Author Contributions}

Luca Ena: conception and design, interpretation of the data, drafting, final approval. Marco Ferrari: acquisition, analysis, and interpretation of the data; revision; final approval. Vittorio Mazzarello, Pasquale Ena: conception and design, acquisition and interpretation of data, revision, final approval.

\section{References}

1 Kim DH, Lee JH, Lee JY, Park YM. Erythema Annulare Centrifugum: Analysis of Associated Diseases and Clinical Outcomes according to Histopathologic Classification. Ann Dermatol. 2016 Apr;28(2):257-9.

2 Baglieri F, Scuderi G. Erythema annulare centrifugum: a "deep type" figurate eruption. G Ital Dermatol Venereol. 2012 Feb;147(1):129-31.

3 McDaniel B, Cook C. Erythema Annulare Centrifugum. In: StatPearls [Internet]. Treasure Island (FL): StatPearls Publishing; 2020.

4 Mid-season risk assessment: seasonal influenza 2015-2016 in the EU/EEA countries. ECDC, 8 Feb 2016. 


\section{Case Reports in Dermatology}

\begin{tabular}{l|l}
\hline Case Rep Dermatol 2021;13:134-140 \\
\hline DOI: 10.1159/000512869 & $\begin{array}{l}\text { @ } 2021 \text { The Author(s). Published by S. Karger AG, Basel } \\
\text { www.karger.com/cde }\end{array}$ \\
\hline
\end{tabular}

Ena et al.: Recurrent Erythema Annulare Centrifugum due to Influenza Type A

5 Ohmori S, Sugita K, Ikenouchi-Sugita A, Nakamura M. Erythema annulare centrifugum associated with herpes zoster. J UOEH. 2012 Sep;34(3):225-9.

6 Piérard GE. [Image of the month: rash of H1N1 influenza]. Rev Med Liege. 2012 Dec;67(12):612-3.

7 Chen JF, Chiang CP, Chen YF, Wang WM. Pityriasis rosea following influenza (H1N1) vaccination. J Chin Med Assoc. 2011 Jun;74(6):280-2.

8 Molina GE, Danesh MJ, Foreman RK, Kroshinsky D. A 78-Year-Old Female with a Diffuse Pruritic Rash and Palmoplantar Desquamation. Dermatopathology (Basel). 2020 Jan;6(4):241-5.

9 Mandel VD, Ferrari B, Manfredini M, Giusti F, Pellacani G. Annually recurring erythema annulare centrifugum: a case report. J Med Case Reports. 2015 Oct;9(1):236.

10 Sardana K, Chugh S, Mahajan K. An observational study of the efficacy of azithromycin in erythema annulare centrifugum. Clin Exp Dermatol. 2018 Apr;43(3):296-9.

11 Meena D, Chauhan P, Hazarika N, Kansal NK, Gupta A. Aceclofenac-Induced Erythema Annulare Centrifugum. Indian J Dermatol. 2018 Jan-Feb;63(1):70-2.
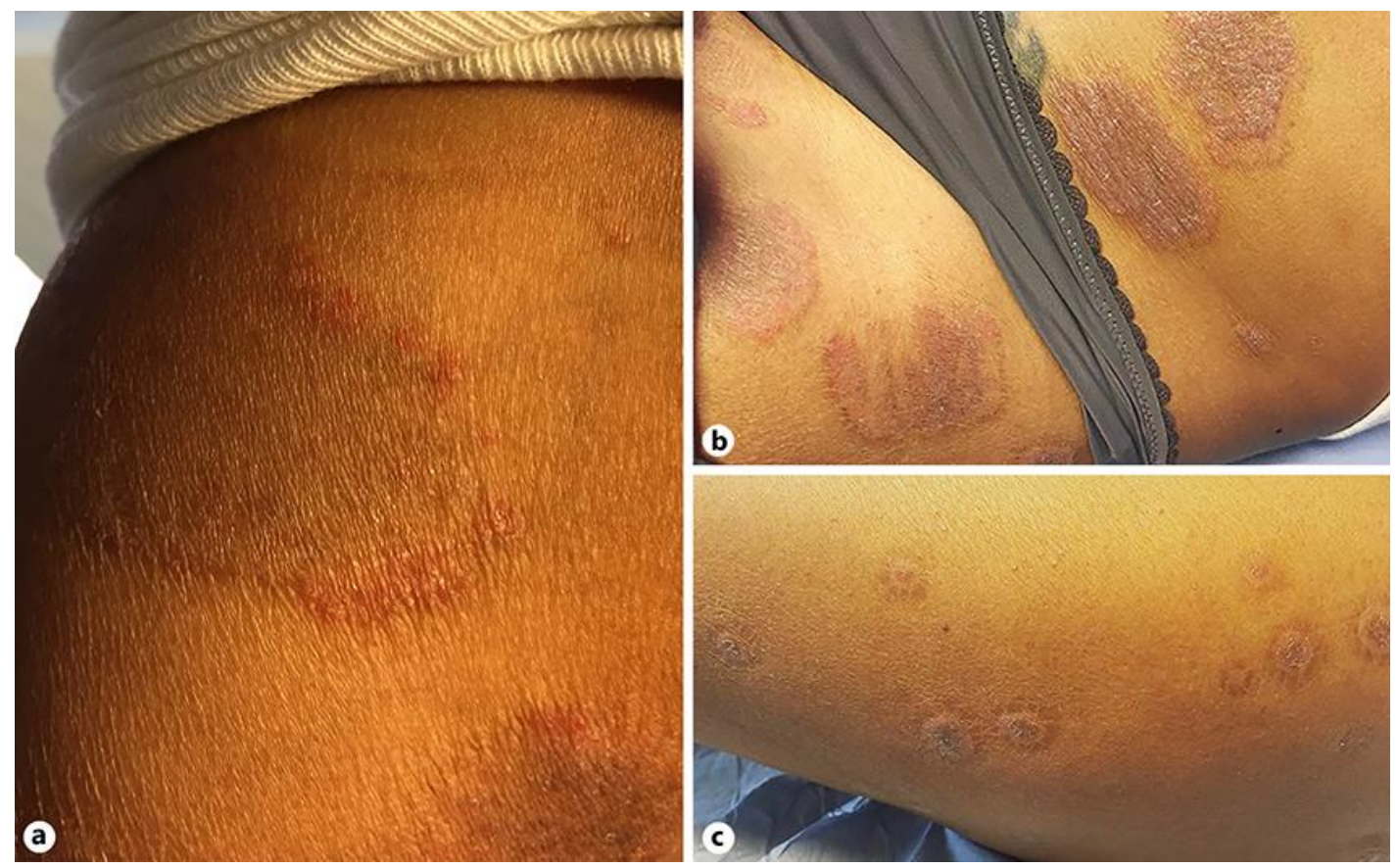

Fig. 1. a, b Clinical presentation of multiple erythematous and violaceous annular plaques involving the forearm and lower abdomen. Some lesions presented a peripheral scaling with an "active" border. c Clinical presentation of a recurrence on the thigh showing multiple and smaller annular lesions. 


\section{Case Reports in Dermatology}

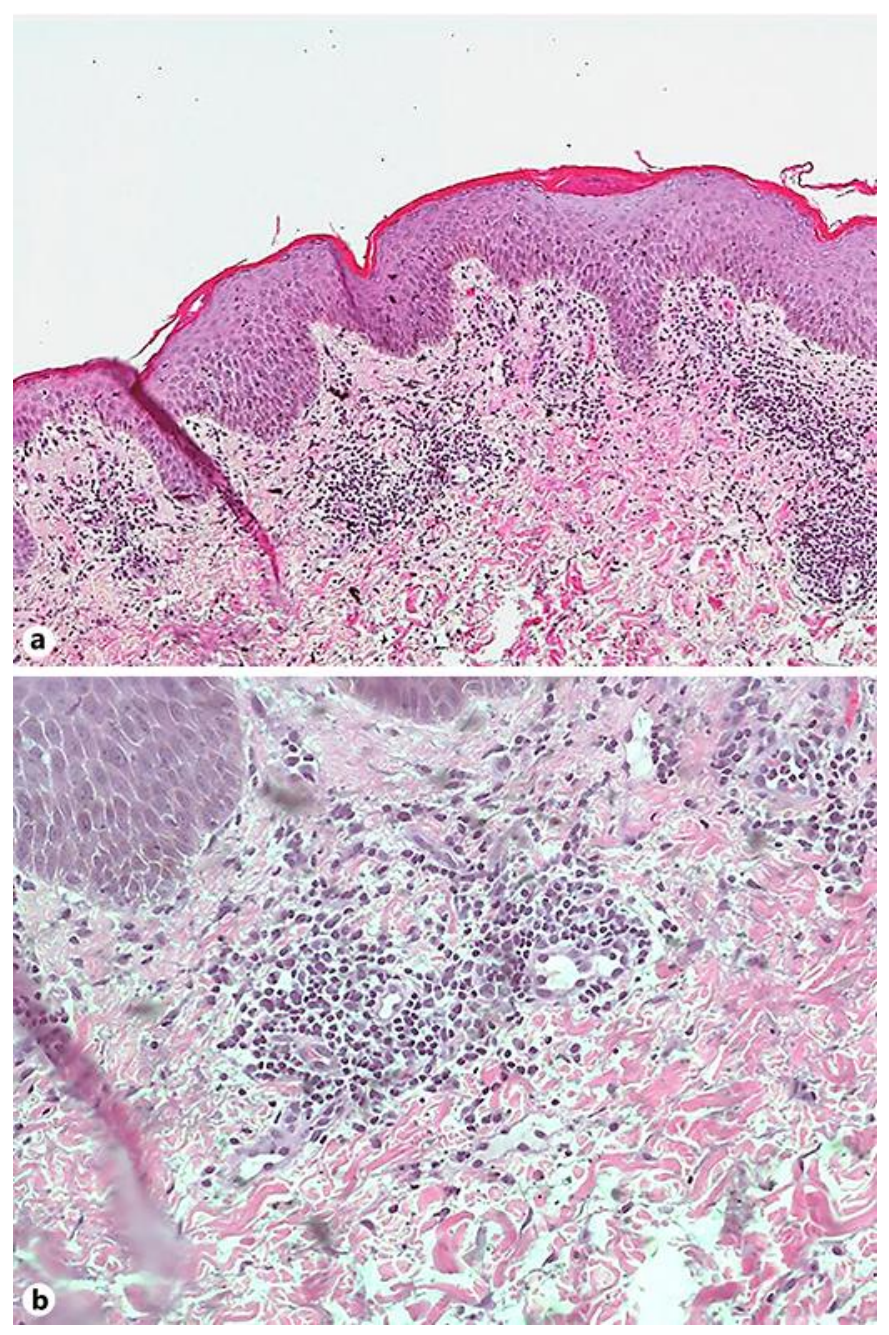

Fig. 2. a, b Histopathological findings of the biopsy showed a moderately intense superficial perivascular lymphohistiocytic infiltrate with epidermal hyperplasia, hyperkeratosis, focal spongiosis, and parakeratosis. Sometimes the perivascular infiltration in the upper dermis results in a "sleeve-like" appearance. Hematoxylin and eosin stain, original magnification: $\times 4(\mathrm{a}), \times 10(\mathrm{~b})$. 
Case Reports in Dermatology
Case Rep Dermatol 2021;13:134-140

DOI: $10.1159 / 000512869$

(c)

(C) 2021 The Author(s). Published by S. Karger AG, Basel www.karger.com/cde

Ena et al.: Recurrent Erythema Annulare Centrifugum due to Influenza Type A

Table 1. Conditions observed in association with erythema annulare centrifugum

Diseases Drugs

Tinea

Rheumatoid arthritis

S. of Sjögren

Thyroiditis

Pregnancy

Cancer (thyroid, ovarian)

Influenza

Herpes zoster
Amitriptyline

Cimetidine

Finasteride

Etizolam

Aldactone

Gold Thiomalate

Aceclofenac

Immunotherapy agent

Ustekinumab

Chloroquine

Hydroxychloroquine 